\title{
Treatment with Hyaluronic Acid Injections in a Patient with Craniosynostosis
}

\author{
Authors: \\ *Daniel Alejandro Muszalski, ${ }^{1}$ Luciana Yacomotti ${ }^{2}$ \\ 1. Department of Dermatology, DermaBaires, Buenos Aires, Argentina \\ 2. Medical Director, GC Skin, Los Angeles, California, USA \\ *Correspondence to consultoriodermabaires@gmail.com \\ Disclosure: $\quad$ The authors have declared no conflicts of interest. \\ Received: \\ 27.05.21 \\ Accepted: \\ 13.01.22 \\ Keywords: \\ Craniosynostosis, fillers, frontal region, hyaluronic acid, temporal region. \\ Citation: \\ EMJ Dermatol. 2022; DOI/10.33590/emjdermatol/21-00118.
}

\section{Abstract}

Case report: A case of a 25-year-old White female left with residual bone deformity after surgical correction of craniosynostosis during childhood is presented here. The significant psychological discomfort from her deformity caused the patient to experience clinical depression and social phobia.

Objectives: The use of soft tissue fillers has grown in popularity for minimally invasive cosmetic procedures. The authors discuss the use of hyaluronic acid (HA) as an available treatment option for the reconstructive volumisation of postsurgical bony deformities in patients with congenital craniosynostosis.

Discussion: Although fat graft techniques have previously been described, to the best of the authors' knowledge, this is the first report of HA fillers as an available treatment option for the reconstructive volumisation of residual bony deformities in patients with craniosynostosis. The negative emotional impact of facial deformities can be dramatically improved by these procedures, making HA a valuable option in providing patients with a highly acceptable cosmetic result.

Conclusions: HA can be successfully used as a non-surgical alternative to correct bone deformities of any aetiology. It is a relatively simple and effective technique that leads to cosmetically acceptable results. The authors emphasise the importance of training to gain an in-depth knowledge of the facial anatomy. Intravascular injections in the forehead and temporal fossa can lead to skin necrosis or blindness, therefore it is imperative to perform safe and proper facial aesthetics injections and to manage possible complications before injections.

\section{INTRODUCTION}

Craniosynostosis is a condition in which one or more sutures in a baby's skull fuse together prematurely, causing growth problems of the brain and skull. Premature closure of the sutures can also cause deformities of the facial bones and skull. Craniosynostosis may arise as part of a genetic syndrome (e.g., Apert, Crouzon, Pfeiffer, Saethre-Chotzen), but it occurs more frequently as an isolated defect.

A full range of surgical options are available for craniosynostosis treatment, from strip craniectomy to whole-vault cranioplasty. The 
main goal of surgery is to reduce intracranial pressure and treat the resulting craniofacial asymmetries. However, the most common complication after craniosynostosis surgery is an incomplete correction of deformities, which can sometimes lead to aesthetic and psychological problems that impact the patient's self-esteem. ${ }^{2}$ It is, therefore, essential to treat these patients using a multidisciplinary approach. ${ }^{3}$ Implementing a long-term assessment is essential to develop additional treatments.

Various tools that restore volume in a defined area to treat residual craniosynostosis deformities have been described, including autologous fat transfer. This technique is minimally invasive and is a good alternative for improving fronto-orbital asymmetries in residual craniosynostosis deformities. ${ }^{4,5}$ Augmentation using the patient's fat, which was first documented in the $20^{\text {th }}$ century, can be employed to replace volume after trauma.

\section{CASE REPORT}

Here, a case of a 25-year-old female with a history of congenital craniosynostosis is reported. The patient underwent corrective surgery at a young age, although her records are not available for review. The frontotemporal bone deformity was the patient's chief complaint. Several plastic surgeons evaluated the patient: none came up with an optimal treatment plan, instead she was referred to various psychiatrists and psychologists. The patient was diagnosed with depression and social phobia and was started on several antidepressant medications. The patient's quality of life was severely impaired, and they ended up having to work from home.

After assessing the patient, the authors came up with a treatment plan consisting of hyaluronic acid (HA) fillers. The treatment was used to improve frontotemporal deformity and was performed in two sessions, 15 days apart. High elastic modulus (G') HA (20 mg/mL) was used in this case, since the thickness of the skin allowed it. An advantage of using high viscosity HA fillers is that they have a longer duration of effect, up to 18 months. However, intermediate or low G' HA (15.0-17.5 mg/mL) is also a good option as the filler can be evenly distributed with a gentle massage.
The authors decided to use needles instead of cannulas for technical reasons, modifying the angle of entry into the skin according to the injection site. Prior injection aspiration was performed at each point to minimise the chance of intravascular occlusion. The supratrochlear and supraorbital foramina were both identified to avoid deep injection near them. The boluses injected were always small in order to reduce the occurrence of inadvertent intravascular injection. A supraperiosteal injection on the temporal region was performed, $1 \mathrm{~cm}$ above the orbital rim on the temporal fusion line and $1 \mathrm{~cm}$ lateral to this point, using the 'one up, one over' technique to identify the safest point when injecting the temporal fossa.

Pre-procedure (Figure 1), immediate post-procedure (Figure 2), and 18 months post-procedure (Figure 3) photographs were then taken.

\section{DISCUSSION}

HA fillers are a promising alternative to consider for the treatment of cranial bone deformities in patients with craniosynostosis and other aetiologies. The use of autologous fat injections for the correction of bone deformities has been previously reported. ${ }^{5}$ Recent data by Coleman et al. ${ }^{6}$ suggest an improved duration of correction with autologous fat transfer. However, it is not clear whether autologous fat is an ideal soft tissue filling agent, particularly given its highly variable duration of correction. Additionally, fat grafting procedures most often involve surgery with some amount of anaesthesia; this approach requires surgical liposuction to harvest adipose tissue and several sessions to achieve the desired results.

Although fat graft techniques have previously been described, to the best of the authors' knowledge, this is the first report of HA fillers as an available treatment option for reconstructive volumisation of residual bony deformities in patients with craniosynostosis. One of the main advantages of HA fillers is their easy reversibility, which is vital in the event of accidental intravascular injection, as hyaluronidase could revert the consequent tissue damage or eventual amaurosis. In this case report, the authors chose a cross-linked, high $\mathrm{G}^{\prime} \mathrm{HA}(20 \mathrm{mg} / \mathrm{mL})$ that can 


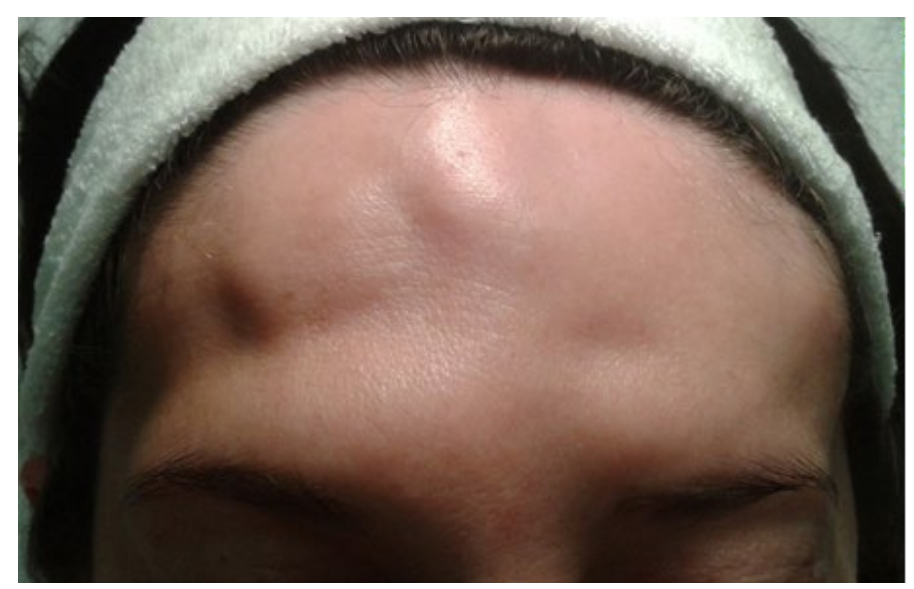

Figure 1: A pre-procedure photograph of the patient.

Bone irregularity at the frontal and temporal levels, with depressions of different depths, are evidenced by the shadows generated.

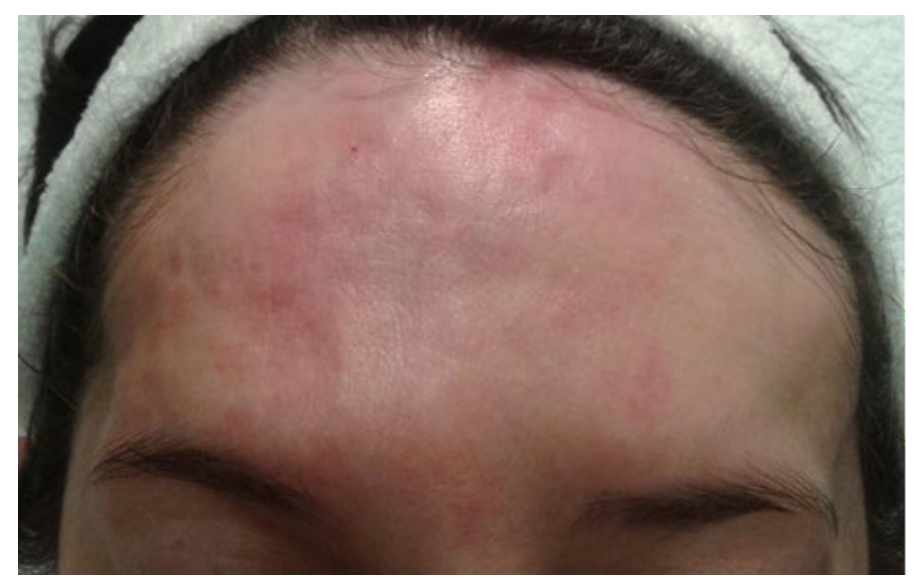

Figure 2: A photograph of the patient taken immediately post-procedure.

An improvement in bone irregularity and depressions is evident, although transient erythema still persisted in the patient due to recent treatment.

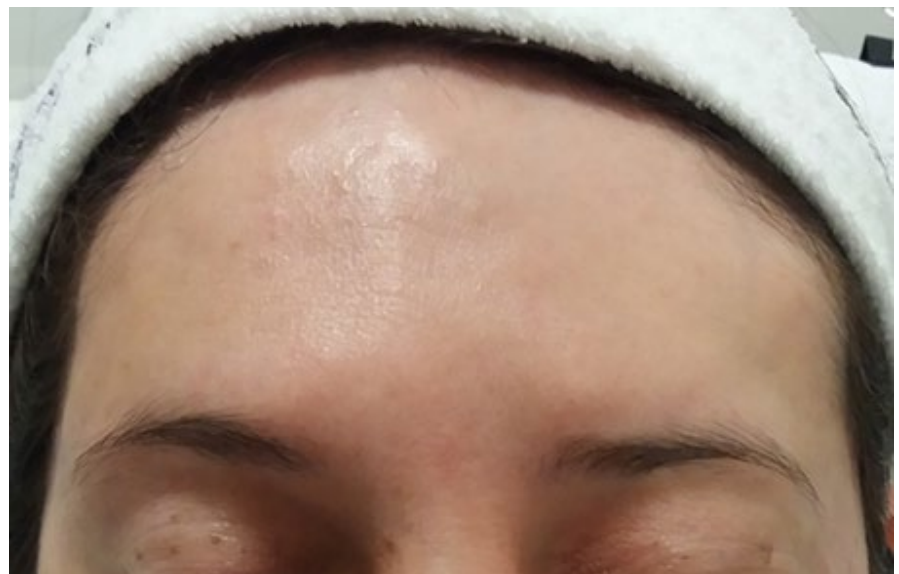

Figure 3: A photograph of the patient taken 18 months post-procedure.

The final result with the disappearance of bone irregularity and depressions can be seen here. 
last up to 18 months. Surprisingly, the correction lasted longer than expected, remaining intact at the 18-month follow-up.

Lazzeri et al. ${ }^{7}$ reviewed 29 articles describing 32 patients with iatrogenic blindness: in 15 patients, blindness occurred after fat injection; in the other 17 patients, it followed the injection of various other materials, including silicone oil, calcium hydroxyapatite, bovine collagen, and HA, with blindness occurring after $\mathrm{HA}$ injections in two out of these 17 patients. $^{7}$ Furthermore, Chatrath et al. ${ }^{8}$ studied a total of 190 cases of filler-induced blindness, the primary cause of which was due to autologous fat injections (90 cases; $47 \%$ ), with the second most common cause attributed to HA injections (53 cases; 28\%). ${ }^{8}$

Goodman et al. ${ }^{9}$ found that accidental intravascular injection, even among very experienced injectors, was high. Although $71 \%$ had 11 years or more of experience, $62 \%$ of these injectors experienced an inadvertent intravascular injection, which reaffirms that anatomical knowledge is essential to minimise these complications. ${ }^{9}$ The authors recommend that injections of the frontotemporal region should be performed by physicians with extensive knowledge of the local anatomy. Intravascular injections and extrinsic compressions are more frequently associated with skin necrosis, and studies have shown that small amounts of filler can occlude wide arterial paths. The equation for the volume of a cylinder $\left(\pi r^{2} h\right)$ tells us that just $0.01 \mathrm{~mL}$ of a product would be enough to fill $5.00 \mathrm{~cm}$ of a $0.05 \mathrm{~cm}$ diameter vessel, assuming that it did not dilate. ${ }^{10}$ Intra-arterial injections in the frontotemporal region, supraorbital artery, supratrochlear artery, or the anterior or frontal branch of the superficial temporal artery can also potentially deliver the filling directly to the ophthalmic artery, and from there to the central retinal artery, resulting in amaurosis. ${ }^{11-13}$

For skin preparation, chlorhexidine plus alcohol was used to achieve the best antibacterial coverage. ${ }^{14,15}$ While the use of cannulas is considered safer for injection, the authors decided to use 27-gauge needles due to bone irregularities in the patient. ${ }^{15-18}$

The needle was directed oblique to the skin (at a $45^{\circ}$ angle) to prevent filler from leaking out through the puncture site and being deposited above the frontal muscle, with the eventual need of removal with hyaluronidase. ${ }^{16}$ After the needle was inserted, the syringe plunger was withdrawn. At least 5 seconds should pass to observe if blood aspirates into the needle hub to reduce the risk of intravascular injection, although this aspiration time is controversial, and many authors recommend waiting 10 seconds. $^{19,20}$ The authors of this study recommend a safety margin of $2 \mathrm{~cm}$ above the supraorbital and supratrochlear foramen (superior orbital border).21,22 The multiple supraperiosteal bolus injection techniques were applied with a small amount of filler $(0.1 \mathrm{~mL})$ as avoiding larger boluses minimises tissue damage in the event of inadvertent intravascular injection or extrinsic vascular compression. Lower volumes injected per point will also significantly decrease the chance of amaurosis by intravascular injection. ${ }^{23}$ Some authors recommend boluses of less than $0.84 \mathrm{~mL}$ in the periocular region, and boluses less than $0.03 \mathrm{~mL}$ in regions close to the supratrochlear foramen. ${ }^{24-26}$ Other authors state that, by injecting aliquots $<0.10 \mathrm{~mL}$ in the facial soft tissues, they can already overcome the entire volume of the supratrochlear artery from the injection site at the glabella to the level of the orbital apex. ${ }^{26}$

Supraperiosteal injection on the temporal region was performed $1 \mathrm{~cm}$ above the orbital rim on the temporal fusion line, and $1 \mathrm{~cm}$ lateral to this point using the 'one up, one over' technique: this is the safest point when injecting the temporal fossa. The needle was inserted perpendicular to the skin, at a $90^{\circ}$ angle down to the periosteum. Prior to injection, the plunger was retracted, and, in the absence of blood, $0.40-0.50 \mathrm{~mL}$ of HA was injected. With the use of the 'one up, one over' technique, the filler injected into the temporal fossa generated a tissue displacement effect instead of diffusion. This injection technique was previously studied on cadavers using CT scans. ${ }^{27}$

\section{CONCLUSION}

The authors believe that, among the currently available filling materials, HA is an excellent option for treating cranial deformities in patients with craniosynostosis. One of the main advantages of HA fillers is the possibility of reversibility in the event of accidental intra-arterial injection. The authors stress that injections using autologous fat, calcium hydroxyapatite, polylactic acid, and 
acrylates are not amenable to this treatment and should be used with extreme caution in high-risk areas, such as those outlined by Beleznay et al. ${ }^{28}$ Finally, the authors highlight the importance of having a deep anatomical knowledge and managing for the eventual complications of injectors before performing these procedures.

\section{References}

1. Pelc A, Mikulewicz M. SaethreChotzen syndrome: case report and literature review. Dent Med Probl. 2018;55(2):217-25

2. Persing JA. MOC-PS(SM) CME article: management considerations in the treatment of craniosynostosis. Plast Reconstr Surg. 2008;121(Suppl 4):1-11.

3. Warren SM et al. Parameters of care for craniosynostosis: craniofacial and neurologic surgery perspectives. Plast Reconstr Surg. 2012;129(3):731-7.

4. Castro-Govea $Y$ et al. Volumetric lipoinjection of the fronto-orbital and temporal complex with adipose stem cells for the aesthetic restoration of sequelae of craniosynostosis. Arch Plast Surg. 2018;45(2):128-34.

5. Rivoalan F. [An oxycephaly deformation corrected with a fat tissue transplant]. Ann Chir Plast Esthet. 2012;57(6):618-21. (In French).

6. Coleman SR. Long-term survival of fat transplants: controlled demonstrations. Aesthetic Plast Surg. 1995;19(5):421-5.

7. Lazzeri D et al. Blindness following cosmetic injections of the face. Plast Reconstr Surg. 2012;129(4):995-1012.

8. Chatrath $\vee$ et al. Soft-tissue fillerassociated blindness: a systematic review of case reports and case series. Plast Reconstr Surg Glob Open. 2019;7(4):e2173.

9. Goodman GJ et al. Experience and management of intravascular injection with facial fillers: results of a multinational survey of experienced injectors. Aesthetic Plast Surg. 2016;40(4):549-55.

10. Townshend A. Blindness after facial injection. J Clin Aesthet Dermatol. 2016;9(12):E5-7.
11. Sykes JM. Applied anatomy of the temporal region and forehead for injectable fillers. J Drugs Dermatol. 2009;8(Suppl 10):s24-7.

12. Loh KT et al. Prevention and management of vision loss relating to facial filler injections. Singapore Med J. 2016;57(8):438-43.

13. Tansatit $\mathrm{T}$ et al. Periorbital and intraorbital studies of the terminal branches of the ophthalmic artery for periorbital and glabellar filler placements. Aesthetic Plast Surg. 2017;41(3):678-88.

14. Bertarini F, Muszalski D. Antisépticos en procedimientos invasivos estéticos y no estéticos. 2020. Available at: https://piel-l.org/blog/48011. Last accessed: 20 January 2022.

15. Diomedi A, et al. [Antiseptics and disinfectants: aiming at rational use. Recommendations of the advisory committee on healthcare associated infections. Sociedad Chilena de Infectología]. Rev Chilena Infectol. 2017;34(2):156-74. (In Spanish).

16. Sundaram $\mathrm{H}$ et al. Blunt-tipped microcannulas for the injection of soft tissue fillers: a consensus panel assessment and recommendations. $J$ Drugs Dermatol. 2012;11(8):s33-9.

17. van Loghem JAJ et al. Cannula versus sharp needle for placement of soft tissue fillers: an observational cadaver study. Aesthet Surg J. 2017:38(1):7388.

18. Funt D, Pavicic T. Dermal fillers in aesthetics: an overview of adverse events and treatment approaches. Clin Cosmet Investig Dermatol. 2013:6:295-316

19. Karimi $\mathrm{K}$ et al. Microcannula utilization for injectable facial fillers: standard of care? American J Cosmet Surg. 2018;35(4):189-97.
20. Casabona G. Blood aspiration test for cosmetic fillers to prevent accidental intravascular injection in the face. Dermatol Surg. 2015;41(7):841-7.

21. Cong LY et al. Topographic analysis of the supratrochlear artery and the supraorbital artery: implication for improving the safety of forehead augmentation. Plast Reconstr Surg. 2017;139(3):620e-7e

22. van Loghem $\mathrm{J}$ et al. Consensus on the use of hyaluronic acid fillers from the cohesive polydensified matrix range: best practice in specific facial indications. Clin Cosmet Investig Dermatol. 2021;14:1175-99.

23. Almeida RT et al. Hyaluronic acid in the rejuvenation of the upper third of the face: review and update. Part 2: temporal and supraorbital regions. Surg Cosmet Dermatology. 2017;9(2):113-21.

24. Woodward JA. Periocular fillers and related anatomy. Cutis. 2016;98(5):330-5.

25. Coleman SR. Avoidance of arterial occlusion from injection of soft tissue fillers. Aesthet Surg J. 2002;22(6):555-7.

26. Khan TT et al. An anatomical analysis of the supratrochlear artery: considerations in facial filler injections and preventing vision loss. Aesthet Surg J. 2016;37(2):203-8.

27. Lamb JP et al. Abstract: threedimensional CT validation of supraperiosteal temple Volumization with hyaluronic acid filler techniques. Plast Reconstr Surg Glob Open. 2018;6(9 Suppl):166.

28. Beleznay $\mathrm{K}$ et al. Avoiding and treating blindness from fillers: a review of the world literature. Dermatol Surg. 2015;41(10):1097-117. 